\title{
émulations
}

\section{Représentation et valeur politique du conflit ethnique en ex-Yougoslavie dans Tactics Ogre}

\section{De Sarajevo à Baramus}

\author{
Sébastien Wit
}

Émulations - Revue de sciences sociales 2019, n 30, "Comment les jeux font-ils société. Contenus, pratiques et médiations ludiques ».

\section{Article disponible à l'adresse suivante}

https://ojs.uclouvain.be/index.php/emulations/article/view/wit

\section{Pour citer cet article}

Sébastien Wit, « Représentation et valeur politique du conflit ethnique en ex-

Yougoslavie dans Tactics Ogre. De Sarajevo à Baramus », Émulations, n³0, Mise en ligne le 3 septembre 2019.

DOI : 10.14428/emulations.030.05

Distribution électronique : Université catholique de Louvain (Belgique) : ojs.uclouvain.be

(c) Cet article est mis à disposition selon les termes de la Licence Creative Commons Attribution, Pas d'Utilisation Commerciale 4.0 International. http://creativecommons.org/licenses/by-nc/4.0/

Éditeur : Émulations - Revue de sciences sociales / Presses universitaires de Louvain https://ojs.uclouvain.be/index.php/emulations

ISSN électronique : 1784-5734

PUL PRESSES 


\title{
Représentation et valeur politique du conflit ethnique en ex-Yougoslavie dans Tactics Ogre
}

\author{
De Sarajevo à Baramus
}

Sébastien Wit ${ }^{1}$

\begin{abstract}
[Résumé] Avec Tactics Ogre: Let Us Cling Together sorti en 1995 sur la Super Famicom de Nintendo, Quest propose un jeu vidéo ambitieux sur le plan narratif, et directement inspiré des guerres ethniques faisant rage en ex-Yougoslavie. L'horreur d'une tuerie potentiellement instrumentalisée comme celle de Sarajevo en février 1994 devrait rendre sa transposition dans le médium ludique particulièrement délicate. Comment transformer un crime de guerre en dispositif narratif ? Dans ces conditions, quel rôle la vérité historique joue-t-elle ? Quel rapport se tisse entre l'actualité et la fiction vidéoludique ? Partant de ces interrogations, il s'agira de voir dans cet article le type de discours que le jeu vidéo est en mesure de tenir sur la question de l'ethnicité, prise à la fois dans sa dimension politique et sociale. Cela nous amènera à interroger la contribution de l'industrie vidéoludique à cet imaginaire du pouvoir socialement partagé sans lequel il n'est pas possible de faire société.
\end{abstract}

Mots-clés : Tactics Ogre, jeu de rôle tactique, Yougoslavie, conflit ethnique

[Abstract] Released in 1995 for the Nintendo Super Famicom, Quest's Tactics Ogre: Let Us Cling Together is an ambitious video game in terms of narrativity. Inspired by the conflict in former Yugoslavia, it discusses the issue of ethnic war. Potentially used as a political tool, a slaughter like the one occurring in Sarajevo in February 1994 should complexify its translation into a video game. How can a war crime become a narrative device? From that perspective, what remains of the historical truth? What is the relationship between political events and video game fiction? Addressing these issues, this paper will study what video games can say about ethnicity, considered as both a social and political debate. This will lead us to see how the video game industry contributes to the constitution of a socially shared imaginary of political power without which it is not possible to make society.

Keywords: Tactics Ogre, Tactical Role-Playing Game, Yugoslavia, ethnic war

\section{Introduction}

De 1991 à 2001, les Balkans sont déchirés par une série de conflits sur le territoire de l'ex-Yougoslavie. Parmi les multiples guerres engendrées suite à la dislocation de la construction nationale qu'avait unifiée le régime de Josip Broz Tito², la période 19921995 coïncide avec ce que l'on nomme généralement la guerre de Bosnie-Herzégovine.

\footnotetext{
${ }^{1}$ Université de Picardie Jules Verne, CERCLL, France.

${ }^{2}$ Josip Broz Tito devient le leader du mouvement communiste yougoslave à partir des années 1937-1939. II restera, jusqu'à sa mort en 1980 (à l'âge de 87 ans), le symbole du régime fédéraliste de Yougoslavie qui était supposé assurer l'unité nationale du pays (Haug, 2012).
} 
Opposant Bosniaques, Serbes et Croates, ce conflit a cristallisé les regards de la communauté internationale en raison du siège de Sarajevo. Plus long siège de l'Europe moderne, cette opération militaire de l'armée serbe contre la capitale bosniaque s'est étendue sur les trois années du conflit. Tandis que les télévisions du monde entier diffusent les images de la bataille de Sarajevo, le jeu vidéo - encore dans les débuts de sa diffusion commerciale - s'empare de ce sujet d'actualité.

Développé par Quest ${ }^{3}$ pour la Super Famicom de Nintendo ${ }^{4}$ après le succès d'Ogre Battle: the March of the Black Queen (1993), Tactics Ogre: Let us Cling Together sort au Japon en octobre 1995. Centré sur la question du conflit ethnique, le jeu se déroule dans le monde fictionnel de l'île de Valeria au sein de laquelle s'affrontent trois groupes ethniques : Galgastan, Bacrum et Walister ${ }^{5}$. Après une période d'unité nationale d'une cinquantaine d'années sous le règne du roi Dolgare, le décès de ce dernier entraîne la résurgence des tensions entre les communautés se partageant l'île. Ne serait-ce qu'à travers cette trame diégétique, apparaît la manière dont Tactics Ogre transpose dans un objet vidéoludique la situation en Yougoslavie depuis la mort de Tito. Avec la disparition du dirigeant socialiste, l'équilibre entre Bosniaques, Serbes et Croates se trouve rapidement mis en péril. Les nationalismes ethniques regagnent de la vigueur, et de nombreux peuples des républiques fédératives yougoslaves revendiquent leur autodétermination. Néanmoins, Tactics Ogre ne se contente pas de reprendre le contexte et les principaux acteurs du conflit. Se déroulant à la fin du chapitre I du jeu, le massacre de Baramus n'est pas sans rappeler celui de Markale de février 1994, cette place de Sarajevo où des civils furent tués sans que l'on sache si cela était le fait des forces serbes ou bien si l'armée bosniaque avait décidé de bombarder son propre peuple afin de pousser l'Organisation du traité de l'Atlantique Nord à intervenir (Burg, Shoup, 2000). Ce n'est qu'au début des années 2000 que la justice internationale a attribué la responsabilité de ces exactions à l'armée serbe (Carlson, 2018). Bien qu'un second massacre ait été perpétré en août 1995, celui-ci est trop proche de la sortie de Tactics Ogre - deux mois plus tard - pour avoir lui aussi joué le rôle de référence externe. L'horreur d'une tuerie potentiellement préméditée comme celle de Sarajevo devrait rendre sa transposition dans le médium ludique particulièrement délicate. Comment transformer un crime de guerre en dispositif narratif ? Dans ces conditions, quel rôle la vérité historique jouet-elle ? Quel rapport se tisse entre l'actualité et la fiction vidéoludique ? Partant de ces interrogations, il s'agira de voir le type de discours que le jeu vidéo est en mesure de

\footnotetext{
${ }^{3}$ Quest Corporation est une société japonaise de jeu vidéo fondée en 1988, principalement connue pour le développement de Tactics Ogre et de la série des Ogre Battle. En 2002, Quest a été racheté par Square Enix.

${ }^{4}$ La Super Famicom (pour "Super Family Computer »), aussi connue sous le nom de Super Nintendo, est une console de salon commercialisée par la firme japonaise Nintendo à partir de 1990. Elle succède à la Famicom, sortie en 1983.

${ }^{5}$ Tout au long de l'article, j'utilise les noms issus de la traduction non-officielle du jeu, diffusée en 2010 par «Aeon Genesis ». Le patch est disponible sur ROMhacking.net. En ligne, consulté le 12 juillet 2019. URL : https://www.romhacking.net/translations/1501. À noter que, dans Tactics Ogre, ces trois noms désignent aussi bien les ethnies que les territoires (parfois étatiques) occupés par ces dernières.
} 
tenir sur la question de l'ethnicité, prise à la fois dans sa dimension politique et sociale. Dans un contexte de redécouverte des valeurs heuristiques de la fiction (CoulombGully, Esquenazi, 2012), cela nous permettra d'examiner la fiction vidéoludique en tant que catégorie de la connaissance, porteuse d'un savoir sur le monde.

\section{Tactics Ogre et le genre du Tactical Role-Playing Game}

\subsection{Le deuxième volet de la saga Ogre Battle}

Dans le paysage du jeu vidéo du début des années 1990, Tactics Ogre constitue une référence incontournable pour le genre du Tactical Role-Playing Game (Tactical RPG). Après le succès du premier jeu de la série Ogre Battle, Quest poursuit son entreprise de renouvellement des codes du jeu vidéo avec ce projet ambitieux, développé pour la Super Famicom. Les références au premier opus de la série y jouent un rôle central. Servant de cadre fictionnel de référence, elles participent pleinement à l'installation de l'intrigue du deuxième jeu. Ainsi, Tactics Ogre commence-t-il par la rencontre entre le protagoniste - Denim - et un groupe de mercenaires de Zenobia, l'un des royaumes au cœur de l'histoire d'Ogre Battle. Par son usage de personnages récurrents, la série s'assure une cohérence et se dote d'une intrigue complexe, dépassant les limites de la seule île de Valeria. Au lendemain de la chute de l'Union des républiques socialistes soviétiques, la complexité géopolitique demeure une donnée essentielle de l'imaginaire des années 1990. Les souvenirs des conflits interposés entre les deux super-puissances sont tenaces, et tout porte à croire que Tactics Ogre profite du monde fictionnel d'Ogre Battle afin de laisser planer, derrière le conflit ethnique de Valeria, l'ombre d'un affrontement entre les deux grands royaumes que sont Lodis et Zenobia 6 .

\subsection{La scène de massacre : point de départ de la bifurcation narrative}

Outre son système de jeu tactique mêlant des déplacements au tour par tour sur un espace isométrique à une gestion des personnages empruntée au jeu de rôle (système qui sera ensuite popularisé par Final Fantasy Tactics), Tactics Ogre est resté célèbre pour sa propension à laisser au joueur le choix entre plusieurs parcours narratifs. Notamment, à la fin du chapitre I, le joueur doit décider - avec Denim - de suivre ou non les ordres du duc Ronway, et de massacrer ou non les réfugiés de la ville de Baramus. Ce choix moral constitue le premier embranchement narratif du jeu, et conduit le joueur à suivre la version « loyale » de l'intrigue ou à s'engager dans la ligne diégétique « chaotique ». Héraut de la résistance de Walister, Denim est confronté à la Realpolitik du duc Ronway, prêt à perpétrer une tuerie de masse à Baramus alors que les réfugiés sont euxmêmes originaires de Walister. Anticipant le refus de ces populations pratiquement

\footnotetext{
${ }^{6}$ À ce titre, la deuxième phase dialoguée du jeu est plus que significative. Le personnage de Vice soupçonne Lodis et Zenobia de vouloir provoquer une guerre de grande ampleur en instrumentalisant la situation politique de lîle de Valeria. Le parallèle avec certains épisodes de la Guerre froide, à l'image de la crise de Cuba, semble s'imposer de manière évidente.
} 
réduites en esclavage de se joindre à la révolte, le duc entend tirer profit d'une scène de purge ethnique pour en faire porter la responsabilité au cardinal Barbatos, le dirigeant de Galgastan. Ainsi, tout Tactics Ogre a pour toile de fond ces errements politiques engendrés par le conflit ethnique. Constamment tiraillée entre pulsions guerrières et aspirations à un avenir pacifié, l'intrigue du jeu se confronte au défi de la représentation des enjeux sociaux, philosophiques et politiques d'une guerre fratricide.

\section{De la Yougoslavie à Valeria : la réalité historique et sa représentation fictionnelle}

\subsection{Médias et mentalités : la loi de l'offre et de la demande ?}

L’influence de la réalité historique sur le développement de Tactics Ogre ne souffre d'aucune ambiguïté. Dans une interview de 19957, Yasumi Matsuno - en charge de la série des Ogre Battle chez Quest - établit un parallèle direct entre l'univers de Valeria et les tensions ethniques entre Serbes, Croates et Bosniaques. Au cours de cet entretien, il développe une vision de la création vidéoludique privilégiant les liens avec l'actualité :

Je pense que ce qui suscite l'intérêt des joueurs varie en fonction de l'air du temps. Par exemple, quand j'ai sorti Ogre Battle, la Guerre du Golfe venait juste d'avoir lieu. C'était un sujet dont on parlait constamment aux informations. Et même si ce n'était pas conscient, ce qui s'était passé pendant la guerre restait gravé dans ma tête. À mon avis, c'est la raison pour laquelle un jeu de simulation de guerre comme Ogre Battle a été aussi bien reçu. Je pense que les personnes qui ont acheté ce jeu s'intéressaient déjà au sujet avant de se le procurer. Dans le cas de Tactics Ogre, j'avais en tête la guerre de Yougoslavie [...]. Même si je ne comprends pas tout, je sais qu'il y a plusieurs groupes ethniques en ex-Yougoslavie et qu'ils se battent entre eux. Tactics Ogre est basé sur une vision du monde très complexe. Si le joueur ne dispose pas de ces connaissances, il risque de ne pas réussir à entrer dans le jeu. Cependant, pour celui qui connaît la situation en Yougoslavie, ce sera un très bon jeu. Avant ces événements, un tel jeu aurait été inenvisageable ${ }^{8}$.

Après avoir rappelé le poids de la guerre du Golfe sur Ogre Battle, Matsuno suggère ici que tout ce qui est véhiculé par les médias d'information s'imprime dans l'esprit du public. « Pays de races et de guerres » (je traduis un autre passage de l'interview), le territoire de l'ex-Yougoslavie apparaît pour Matsuno comme un terreau fertile afin de favoriser la réception d'un jeu vidéo narrativement complexe. Dans Tactics Ogre, aucun manichéisme ne vient séparer les bons des méchants. Comme l'énonce le personnage

\footnotetext{
7 L'interview en question accompagnait la version Super Famicom de Tactics Ogre. Elle a été republiée en 1997 dans le no 17 (août 1997) du Dengeki Super Famicom lors de la sortie du jeu sur la PlayStation de Sony. Une version peut être trouvée en ligne via l'Internet Archive de I'URL : http:// minic.com/ogre/info/ogredata_03.html (consulté le 23 juillet 2017).

${ }^{8} \mathrm{Je}$ traduis ici l'interview publiée en japonais, en m'aidant partiellement de la traduction en anglais disponible sur le site gamefaqs.com. En ligne, consulté le 9 décembre 2018. URL : https://gamefaqs. gamespot.com/boards/999440-tactics-ogre-let-us-cling-together/57892950.
} 
de Canopus au début du chapitre in de la route « Chaos », ce sont les vainqueurs qui font l'histoire. Bourreaux ou victimes, tout n'est qu'une question de point de vue. Cette reprise du topos benjaminien d'une fabrique de l'histoire (Benjamin, 2013 [1940]) nous rappelle que Tactics Ogre s'inscrit dans un moment épistémique de crise de l'histoire. Le récit historique n’est pas la vérité, il n’existe pas de déroulement linéaire des événements (Foucault, 1969). Dans un souci de complexité, Matsuno veut que le monde de Tactics Ogre donne à voir - et à jouer - les jeux de pouvoir (cachés) à l'origine des conflits. Néanmoins, craignant de perdre le joueur en raison du manque de transparence de l'univers fictionnel, Matsuno fait le postulat que la connaissance - ou, disons, la sensibilisation - aux modalités du conflit yougoslave facilitera l'accès au jeu.

D'un point de vue théorique, la thèse de Matsuno est plus que contestable. En établissant un parallèle entre le succès d'un jeu de simulation de guerre (ici Ogre Battle) et les relais médiatiques de la Guerre du Golfe, Matsuno présuppose un lien causal entre l'actualité et la réception d'un genre vidéoludique. Il ne s'agit pas simplement du fait que l'actualité rende un sujet familier, ou que la connaissance de celle-ci facilite la prise en main d'un jeu s'en inspirant. Ogre Battle est commercialisé à un moment où la Guerre du Golfe appartient déjà au passé. En revanche, à en croire Matsuno, un je-ne-sais-quoi de cet événement historique aurait subsisté, pour ainsi dire gravé dans les consciences de la société japonaise ; et ce serait ce reliquat mental de l'actualité internationale des dernières années qui aurait motivé, chez les consommateurs, l'achat d'un jeu appartenant au genre de la simulation de guerre. Bien que Matsuno ne développe pas explicitement le même parallèle entre le genre du Tactical RPG et la guerre en ex-Yougoslavie (au moment de l'interview, rien n'assurait encore le succès commercial de Tactics Ogre), le fil de son raisonnement montre clairement qu'il considère les deux jeux selon une même perspective. Or, postuler une telle mécanique - bien huilée - entre l'actualité, l'acte d'achat et l'engagement du joueur (aussi bien sur le plan du gameplay que de la narration) revient en réalité à considérer qu'il existe un imaginaire préétabli traversant de manière inconditionnée l'ensemble de la société. Hors de toutes considérations sociopolitiques, par la seule action subliminale des médias, les individus deviendraient des cibles marketing homogènes ${ }^{9}$; ce dont, faute de preuve, il est permis de douter. Toutefois, le rapport analogique qu'esquisse Matsuno entre les préoccupations d'une époque et le game design présente un véritable intérêt pour la critique. Cela révèle, chez les développeurs du début des années 1990, le souci de formaliser un discours méta capable d'articuler à la fois l'intuition d'une demande sociale et le processus de conception des objets vidéoludiques. C’est cette volonté de penser, à travers le jeu vidéo, un imaginaire partagé qu'il s'agira d'examiner dans la suite de cet article.

\footnotetext{
${ }^{9}$ En 2011, dans une interview au site 4Gamer.net à l'occasion du portage de Tactics Ogre sur la PlayStation Portable de Sony, Matsuno présente explicitement le genre du Tactical RPG comme un genre de niche, motivé par une visée marketing. D'une manière intéressante, toute mention à l'ex-Yougoslavie disparaît au profit d'une conception purement commerciale du jeu vidéo. En ligne, consulté le 23 juillet 2018. URL : http://www.4gamer.net/games/116/G011621/20110426090/.
} 


\subsection{Valeria, un laboratoire politique}

Au début des années 1990, l'intérêt du Japon pour la situation de l'ex-Yougoslavie n’a rien d'étonnant. Bien au contraire, en dépit de leurs divergences idéologiques, les deux pays entretiennent de bonnes relations depuis la fin de la Seconde Guerre mondiale. Des accords sont signés dès les années 1950. En 1968, Tito est accueilli à Tokyo ; puis en 1976 ce sont des membres de la famille royale nippone qui se rendent en Yougoslavie (Glisic, 2016). En pleine Guerre froide, ces relations bilatérales avec un pays communiste sont un moyen pour le Japon de s'émanciper du joug américain. Écrasé sous le poids de la lutte entre les deux blocs, l'archipel nippon partage une communauté de destin avec le régime de Tito, ce dernier ayant cherché dans le mouvement des non-alignés un moyen de tracer sa propre voie (Glisic, 2016).

La réalité historique des rapports diplomatiques entre les deux pays peut expliquer l'image du roi Dolgare dans Tactics Ogre. Récemment décédé, cet homme du peuple devenu un grand monarque avait réussi à unifier les trois groupes ethniques de Valeria. Alors que son règne avait coïncidé avec cinquante années de paix et de prospérité, sa disparition - sans héritier - révèle les dissensions persistant au sein de la société de l'île. Directement inspiré de Tito, Dolgare cristallise une imagerie du pouvoir et de la révolution socialiste, entre fascination et rejet. Comme l'assène Vice dans Tactics Ogre, en dépit de tous les vœux pieux des idéalistes tels que Dolgare, l'égalité n'existe pas. L'homme est naturellement méchant et en quête de soumission. Lans Tartare ne se prive pas de le rappeler à son alter ego, le paladin Lans Hamilton, au début du chapitre Iv. Certains hommes veulent être dominés par un chef. Dans les deux cas, l'allusion à l'idéologie autogestionnaire de la Yougoslavie des années 1950-1960 est patente. Avant le retour en arrière des années 1970, le régime de Tito avait essayé d’imposer l'absence de hiérarchie au sein des entreprises (Samary, 1988). Sous couvert de feintise ludique, ce lieu de suspension des jugements moraux (Lavocat, 2016), Tactics Ogre renvoie à la société les rêves, les espoirs et les craintes qu'elle entretient envers le pouvoir politique. Comme l'a montré Frédérique Leichter-Flack (2015), dans les fictions contemporaines (et notamment celles qui sont à destination de la jeunesse), la question politique prend la forme du « qui vivra qui mourra ». Choisir avec Denim de sacrifier ou non des innocents à Baramus, c'est expérimenter les possibles de la décision politique. Par la réflexion morale et sociale qu'il suscite, le massacre est un laboratoire des cas de conscience.

\section{Entre jeu et récit : le savoir politique de la fiction vidéoludique}

\subsection{L'espace du jeu : vérité historique et savoir politique}

Contrairement à des jeux vidéo faisant de conflits récents le lieu de l'axiomatique ludique (Marti, 2018), Tactics Ogre n'entretient qu'un rapport distant avec la réalité historique. Transposé dans un univers médiéval fantastique, le conflit yougoslave se 
réduit à un paradigme narratif. Le divertissement prend résolument le pas sur la vérité historique, ce qui évite au jeu de se trouver pris dans des polémiques comme celle de 2014 sur Assassin's Creed Unity d'Ubisoft (Martin, Turcot, 2015). Tactics Ogre peut alors s’inspirer du massacre de Merkale en tant que ressort diégétique, sans susciter de problèmes d'ordre éthique. Déplacé dans un monde fictionnel totalement indépendant de la réalité, le massacre de Baramus n'est qu'un reflet lointain de la Sarajevo historique. Pour le joueur, la frontière entre fait et fiction apparaît dans toute sa netteté ${ }^{10}$. Malgré les nombreuses références à la réalité du début des années 1990, la fictionnalité du jeu l'emporte sur les phénomènes de référentialité. Cadré en dehors de la réalité, Tactics Ogre échappe au primat de la vérité historique (d'autant plus lorsque le jeu se termine sur un combat contre l'esprit diabolique, revenu d'entre les morts, de Dolgare).

Le fait que la fictionnalité d'une œuvre dépende de son cadrage a déjà bien été analysé par la critique (Caïra, 2016). Néanmoins, dans le cas de Tactics Ogre, à la faveur de ce cadrage fictionnel sans ambiguïté apparente, le jeu multiplie les incartades aux frontières de la fiction. Lorsque la narration du début du chapitre iv affirme que les conflits ne répondent jamais à des considérations ethniques ou idéologiques mais résultent simplement de la cupidité des dirigeants, elle transforme le dernier acte du jeu en un moment de constitution d'un savoir politique explicite. La guerre de Valeria n'est pas une guerre de races, mais une guerre de classes, opposant paysans et dirigeants. Par son caractère marxisant, ce discours politique détonne en venant substituer la question sociale à celle de l'unité ethnique. Il s'agit là d'un retournement scénaristique majeur dans la mesure où la trame narrative de la fin du jeu rompt avec la grille de lecture des chapitres précédents. Néanmoins, ce choix des développeurs s'explique en partie pour des raisons pragmatiques. Les bifurcations narratives de Tactics Ogre ne disposent pas chacune de leur propre fin. En vérité, les trois versions du chapitre III convergent toutes vers le même chapitre iv, alors que l'allégeance de Denim envers les factions de Valeria diffère à l'intérieur de chacun des segments narratifs. Il était donc nécessaire d'évacuer la question ethnique du dernier chapitre du jeu afin de ne pas rompre la cohérence de l'ensemble. Il n'y a d'ailleurs pas lieu de s'étonner de la mobilisation d'un tel artifice narratif. On sait que les œuvres vidéoludiques démontrent régulièrement une polyphonie des lectures idéologiques, qui se superposent à divers niveaux du game design (Genvo, 2005).

Sans faire de Matsuno un cryptomarxiste, il est tout de même intéressant de mettre en perspective l'idéal de l'unité ethnique dans Tactics Ogre et la réalité socioculturelle du Japon. Traditionnellement, le Japon est traversé par le mythe d'une population monolithique. L'exotisme de l'univers médiéval européen est renforcé par l'invisibilisation

\footnotetext{
10 D'ailleurs, si la presse spécialisée reconnaît bien les motifs du conflit ethnique yougoslave dans la composition de Tactics Ogre, elle met rapidement ce modèle historique à distance en le considérant comme une inspiration anecdotique. Pour exemple, on pourra se référer au compte-rendu de Brendon Taylor sur hardcoregaming101.net. En ligne, consulté le 24 juillet 2018. URL : http:// www.hardcoregaming101.net/tactics-ogre/. Ou encore à celui de Bryan Grosnick sur rpgfan.com. En ligne, consulté le 24 juillet 2018. URL : http://www.rpgfan.com/reviews/Tactics_Ogre_Let_Us_ Cling_Together/index.html.
} 
de la question ethnique dans le discours politique japonais. Après une ouverture aux langues et cultures minoritaires dans les années 1960, le Japon connaît un retour de la doctrine du tan'itsu minzoko kokka (littéralement « l'État-nation mono-racial ») dans les années 1980 (Weiner, 2004). Emmenant le joueur dans le territoire ethnicisé à outrance qu'est Valeria (du moins jusqu'au chapitre Iv du jeu), Tactics Ogre le confronte - via la fiction vidéoludique - à des problématiques politiques particulièrement sensibles. Bien que, par son cadrage, la fiction désamorce le transfert direct de ce discours à la réalité (Fortin, Trémel, 2005), il n'en reste pas moins que le jeu renvoie un imaginaire du pouvoir. Si, comme l'écrit Maurice Godelier (2015), le politique est le fruit d'un imaginaire partagé, alors la mise en représentation d'une imagerie politique (en tant qu'ensemble des figures composant un imaginaire) dans le jeu vidéo contribue pleinement à « faire société ».

\subsection{Le spectacle de la violence : de la scénographie au système d'explication}

Dans l'expérience de jeu de Tactics Ogre, le massacre de Baramus correspond à la première confrontation du joueur avec une scène de violence explicite. La scène donne d'ailleurs lieu à une cinématique (identique, quel que soit le choix de Denim) particulièrement soignée pour la Super Famicom (figure 1).

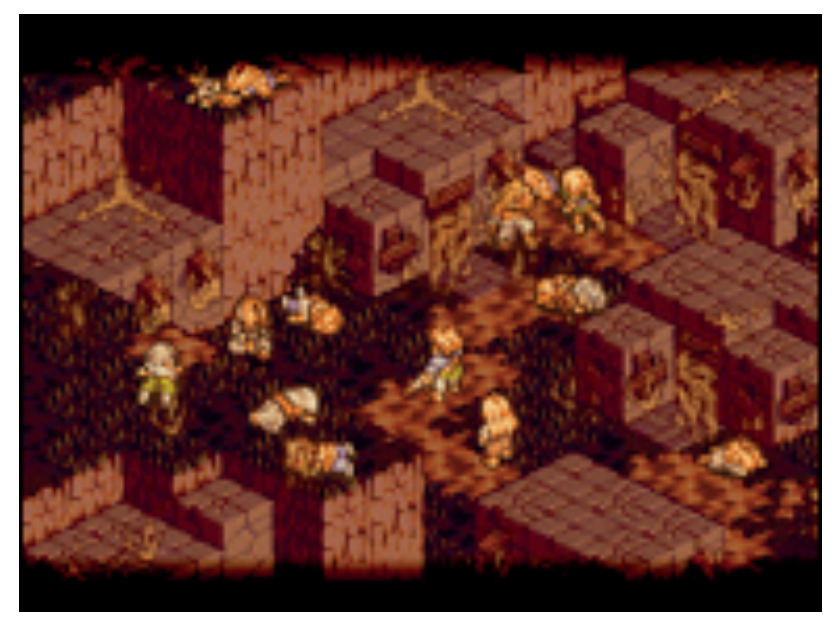

Figure N ${ }^{\circ} 1$ : Fin du chapitre I de Tactics Ogre (Quest, SuperFamicom), tous drolts réservés.

L'épée ensanglantée à la main, le protagoniste se tient devant la ville en proie aux flammes tandis que les cadavres de civils jonchent le sol. Il s'agit là d'une première monstration de violence qui s'intègre dans une fresque morbide plus large : le massacre des habitants de Rime dans le chapitre II, la décapitation du cardinal Barbatos dans la route « Chaos » du même chapitre (le dirigeant de Galgastan est guillotiné devant une foule avide de vengeance), la pendaison de Vice (fin de la version "Chaos » du chapitre III), etc. Ces temps de représentation de la mort scandent le déroulement de l'intrigue, de la même manière que dans les tragédies les plus sanglantes. À la suite 
de ce qu'écrit Friedrich Nietzsche dans la Naissance de la tragédie (2015 [1872]), il est communément admis de faire du spectacle de la violence un invariant du théâtre occidental. Avec des pièces telles que le Titus Andronicus de Shakespeare ou la Médée de Corneille (pour ne citer que ces deux exemples célèbres), l'art dramatique s'est pleinement confronté à la question de la valeur sociale du spectacle de la violence. Régi par la conception aristotélicienne de la purge, le théâtre baroque légitime sa mise en scène des atrocités via son inscription dans une vision partagée de l'art et du social (Biet, Fragonard, 2010). À l'époque contemporaine, l'analyse du savoir politique du jeu vidéo - en l'occurrence japonais - requiert de mettre au jour le système explicatif auquel il s'adosse. Comme l'écrit Bertrand Gervais au sujet des événements du 11 Septembre : «La violence ne signifie pas en soi, elle acquiert une signification par son insertion dans une structure explicative, qu'elle soit sujétale, sociale, philosophique, anthropologique, etc. » (2011 : § 3). Reconstituer le paysage mental du Japon du milieu des années 1990 représente un travail en soi, dépassant le simple cadre de cet article. Néanmoins, le fait de reconnaître dans l'objet vidéoludique une capacité à unir une communauté (nationale ou non) autour d'une violence à expliquer, au même titre que la littérature ou le cinéma, revient à le sortir de l'anomie interactive dans laquelle il se trouve encore parfois enfermé. À considérer le jeu vidéo uniquement sous l'angle d'un système régi par des règles, on en vient parfois à occulter la part humaine de l'activité ludique (Sicart, 2011). La fiction vidéoludique ne se réduit pas à un échange d'input et d'output entre l'humain et la machine. L'interaction ne consiste pas en un simple mécanisme. Elle est sous-tendue par un ensemble de valeurs et de représentations socialement constituées. C’est insérée dans un tel nomos que la fiction devient signifiante et révèle les formes de commun, de vivable qu'elle porte en elle (Besson, 2015).

\subsection{Axiomatique ludique et forme de savoir politique}

Dans le grand débat entre ludologie et narratologie, la dimension interactive du jeu vidéo a pu être utilisée afin de dissocier clairement le jeu du récit. Étant action, le jeu vidéo revêt un caractère normatif - celui des règles du jeu - qui le sépare de la simple force de proposition des récits traditionnels (Lavocat, 2016). Cependant, dans sa généalogie, le Tactical RPG emprunte aux wargames son goût pour le conflit d'envergure, mêlant un grand nombre de paramètres différents (Peterson, 2012). Dans la mécanique de jeu de Tactics Ogre, outre le fait que chaque unité soit identifiée par son ethnie d'appartenance, ainsi que l'organisation politique à laquelle elle a prêté allégeance, le jeu comporte un système de loyauté. En raison de ce dernier, chaque unité est susceptible de quitter l'armée du joueur si les actions de Denim vont à l'encontre des intérêts de l'ethnie d'origine de l'unité. Parallèlement, Denim dispose de son propre compteur mesurant sa réputation avec chacune des ethnies représentées dans le jeu (compteur désigné généralement sous l'appellation «Chaos frame »11. D’un point de vue computationnel, toutes ces données sont cachées au joueur. Celui-ci ignore que les réponses

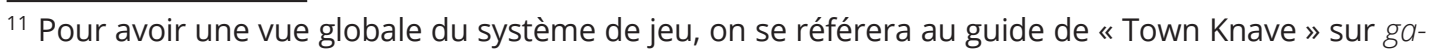


à telles ou telles questions au fil du jeu vont potentiellement avoir un impact sur le cours de sa partie. Comme souvent, l'interactivité entre l'humain et le système de jeu est limitée ; sauf lorsque le joueur décide de rechercher, de lui-même, le mécanisme procédural à l'œuvre (Bogost, 2007). Dans le cas de Tactics Ogre, ce redoublement de la trame narrative par une rhétorique procédurale transforme la question de l'ethnicité en un mécanisme ludique, et aboutit à la mise en scène d'une vision tripartite du pouvoir : le chef (toujours seul, si l'on en croit les commentaires qui apparaissent lorsque l'on appuie sur la touche Select), les troupes (au moral fluctuant) et ce que le jeu nomme "l'idée ", c'est-à-dire ce pour quoi le protagoniste emmène ses hommes. Uniquement présente en creux, cette philosophie politique exprime une manière de faire société - certes implicite pour le joueur, mais au fondement de sa progression dans le jeu. Si la capacité à remonter jusqu'au code constitue véritablement une compétence du joueur (Bogost, 2007), c'est bien par son axiomatique que Tactics Ogre propose la clé d'une forme d'unité sociopolitique au sein de l'̂lle de Valeria. Et le savoir politique ainsi modélisé appartient alors en propre aux caractéristiques du médium vidéoludique.

\section{Conclusion}

Avec Tactics Ogre: Let Us Cling Together, Quest illustre la manière dont la fiction vidéoludique peut traiter la violence - fratricide - de la guerre ethnique. Par le biais d'une narrativité à la complexité assumée, le jeu vidéo donne à voir un certain imaginaire du monde et de la politique. Certes, dans les productions fictionnelles de divertissement, il est toujours délicat de postuler l'appréhension par le public d'un message à consonance politique. Ce n'est pas parce qu'une œuvre traite d'un thème, qu'elle vise obligatoirement à faire réfléchir son destinataire sur le thème en question. Comme le note très justement Sébastien Genvo (2012), tous les jeux, et plus particulièrement les jeux commerciaux (dont Tactics Ogre fait partie), ne disposent pas d'un important potentiel expressif. Ce serait surinterpréter le jeu développé par Quest que de voir en lui une tentative de traiter une problématique historique à travers le cas du conflit ethnique. Cependant, en dépit de ce manque d'expressivité (au sens où l'entend Genvo), Tactics Ogre procède tout de même d'une forme de savoir politique, et ce à un triple niveau. D'un côté, du point de vue de l'anthropologie politique, la mise en scène et le questionnement d'un certain imaginaire du pouvoir s'inscrit de facto dans une perception de ce qui fait société. Un groupe humain ne constitue jamais en soi une société. Il a toujours besoin de se doter d'une autonomie institutionnelle capable de transcender les générations : autrement dit de "faire société » (Thériault, 2007). La locution faire société désigne ainsi le processus social de dépassement de la relation utilitaire du regroupement entre individus afin de jeter les fondements d'une identité commune, d'un imaginaire partagé de l'institution sociopolitique. Que le discours d'un jeu conforte ou jette le doute sur le système politique et idéologique en place, par son intermédiaire

mefaqs.com. En ligne, consulté le 25 juillet 2018. URL : https://gamefaqs.gamespot.com/snes/588770tactics-ogre-let-us-cling-together/faqs/32399. 
tout joueur interagit systématiquement - qu'il le veuille ou non - avec cet imaginaire institutionnel. D’un autre côté, dans son rapport à l'actualité médiatique, le jeu vidéo cristallise des phénomènes d'interculturalité. Par le regard qu'il porte sur un ailleurs temporel, géographique et/ou politique (dans ce cas, l'ex-Yougoslavie du début des années 1990 transformée en univers médiéval fantastique), le jeu vidéo renvoie à la société l'image qu'elle se fait de la question sociopolitique. Enfin, dans la mesure où il se joue, c'est à travers la rhétorique procédurale de son gameplay que l'objet vidéoludique permet à son joueur de vivre - en acte - une certaine conception de la politique, une certaine manière de faire société.

Tactics Ogre ne défend aucunement une position sur le conflit ethnique. Difficile, à l'issue du jeu, de savoir s'il est ou non possible d'éviter ces luttes fratricides, ou de déterminer à qui profite le crime. Le maître mot de Tactics Ogre demeure - comme le dit Matsuno - l'idée de complexité. L'univers est un système dynamique au sein duquel rien n'est réductible à des oppositions binaires. Le joueur est mis en face d'un foisonnement narratif dont il doit rétablir les chaînons manquants. La rhétorique procédurale d'un jeu ne vise pas nécessairement la transmission d'un message. Les jeux à faible valeur persuasive peuvent se contenter de faire du système de jeu une matrice d'exploration d'un espace ludico-narratif (Bogost, 2007). Par l'intermédiaire de la jouabilité d'un monde ludoformé se voulant - pour reprendre les présupposés de Matsuno - l'actualisation de structures d'un imaginaire socialement partagé, existe déjà une forme minimale d'enthymème (Bogost, 2007). Tout système ludique est une case manquante à laquelle se confronte le joueur, un espace intermédiaire au sein duquel peut être ébauché un discours sur ce que le jeu donne à voir (Genvo, 2012).

Au bout du compte, Tactics Ogre ne peut dire quelque chose sur la réalité que dans la mesure où, au milieu de ce triple dispositif (anthropologique, médiatique, procédural), l'histoire et ses événements deviennent un théâtre de références (certes distantes) qu'il revient au joueur d'identifier. Le repérage de la référentialité est la condition du décryptage du travail sur l'imaginaire auquel Matsuno entendait se livrer avec Tactics Ogre. Et c'est précisément pour cette raison que, parmi les fins alternatives du jeu (dépendant du déroulement du chapitre iv), celle de l'assassinat de Denim par un membre d'un groupe radical, brandissant un pistolet (arme pourtant absente du jeu !), est conçue afin de nous rappeler une autre Sarajevo historique (figure 2). 


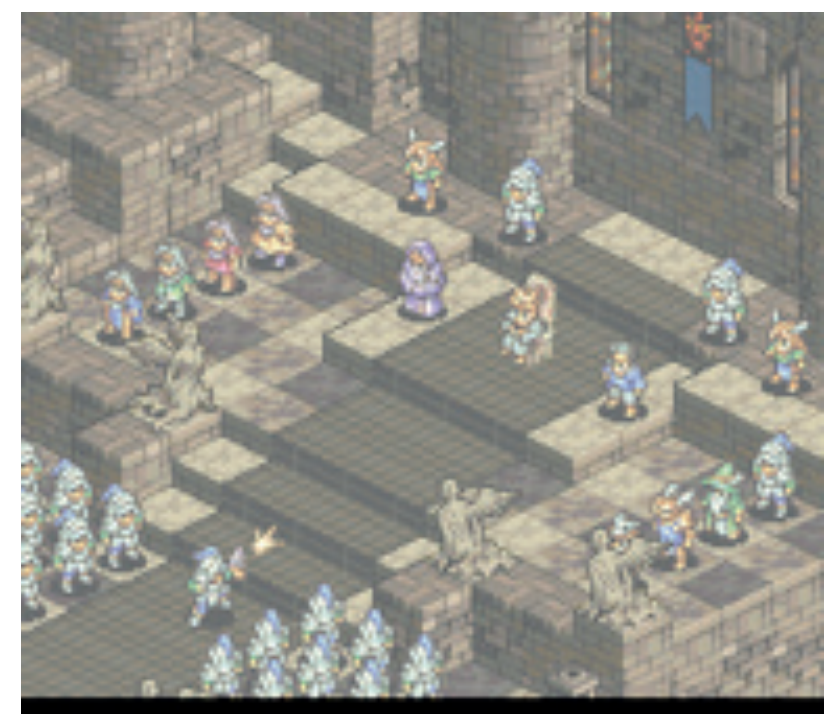

Figure n² 2 : Fin de Tactics Ogre si Kachua est morte (Quest, SuperFamicom), tous droits réservé

\section{Bibliographie}

Benjamin W. (2013 [1940]), Sur le concept d’histoire, Paris, Payot \& Rivages (" Petite bibliothèque Payot »).

Besson A. (2015), Constellations. Des mondes fictionnels dans l'imaginaire contemporain, Paris, CNRS Éditions.

Biet C., Fragonard M.-M. (2010), « Représentation, hyper-représentation et performance des violences politiques et religieuses (mi-xvie / mi-xvire siècle) : théâtre, littérature et arts plastiques », Littératures classiques, vol. 3, $\mathrm{n}^{\circ}$ 73, p. 5-15.

Bogost I. (2007), Persuasive Games. The Expressive Power of Videogames, Cambridge/ Londres, The MIT Press.

Burg S. L., Shoup P. S. (2000), The War in Bosnia: Ethnic Conflict and International Intervention, Armonk, M. E. Sharpe.

Caïra O. (2016), «Théorie de la fiction et esthétique des jeux », Sciences $d u$ jeu, n 6. En ligne, consulté le 24 juillet 2018. URL : https://journals.openedition.org/sdj/671.

Carlson K. B. (2018), Model(ing) Justice: Perfecting the Promise of International Criminal Law, New York, Cambridge University Press.

Coulomb-Gully M., Esquenazi J.-P. (2012), « Fiction et politique : doubles jeux », Mots. Les langages du politique, vol. 2, n 99, p. 5-11.

Fortin T., TRÉMEL L. (2005), « Les jeux de "civilisation” : une représentation du monde à interroger », in T. Fortin, P. MORA, L. TRÉMEL (dir.), Les jeux vidéo : pratiques, contenus et enjeux sociaux, Paris, L’Harmattan (« Champs visuels »), p. 123-167.

Foucault M. (1969), L’Archéologie du savoir, Paris, Gallimard (« Bibliothèque des sciences humaines »). 
Genvo S. (2005), « Approches des jeux vidéo : jugements de valeurs et idéologies », in S. Genvo (dir.), Le game design de jeux vidéo. Approches de l'expression vidéoludique, Paris, L’Harmattan (« Communication et Civilisation »), p. 97-114.

Genvo S. (2012), "Comprendre et développer le potentiel expressif », Hermès. La Revue, No 62.1, p. 127-133. En ligne, consulté le 10 décembre 2018. URL : https://www.cairn. info/revue-hermes-la-revue-2012-1.htm-page-127.htm.

Gervais B. (2011), « Déjouer le spectacle de la violence. Représenter les événements du 11 septembre $2001 »$, E-rea, n 9.1. En ligne, consulté le 25 juillet 2018. URL : http:// journals.openedition.org/erea/1944.

GLISIC J. (2016), « Balancing among Superpowers: Japan-Yugoslavia Relations during the Cold War », Journal of International and Advanced Japanese Studies, vol. 8, p. 145-156.

Godelier M. (2015), L’imaginé, l’imaginaire el le symbolique, Paris, CNRS Éditions.

Haug H. K. (2012), Creating a Socialist Yugoslavia. Tito, Communist Leadership and the National Question, Londres/New York, I.B. Tauris.

Lavocat F. (2016), Fait et fiction. Pour une frontière, Paris, Seuil (« Poétique »).

LeICHTER-FLACK F. (2015), Qui vivra qui mourra. Quand on ne peut pas sauver tout le monde, Paris, Albin Michel.

MARTi M. (2018), « L'Histoire dans le jeu vidéo, une généalogie narrative problématique ? Le cas de la guerre d'Espagne (1936-1939) et de sa ludicisation », Sciences du jeu, n 9. En ligne, consulté le 24 juillet 2018. URL : http://journals.openedition.org/sdj/1041.

Martin J.-C., TuRcot L. (2015), Au cour de la Révolution. Les leçons d'histoire d’un jeu vidéo, Paris, Éditions Vendémiaire (« Chroniques »).

NieTzsche F. (2015 [1872]), Naissance de la tragédie, Paris, Flammarion.

Peterson J. (2012), Playing at the World: A History of Simulating Wars, People and Fantastic Adventures, from Chess to Role-Playing Games, San Diego, Unreason Press.

SAMARY C. (1988), Le Marché contre l'autogestion. L'expérience yougoslave, Malakoff, Publisud/La Brèche.

SiCART M. (2011), « Against Procedurality », Game Studies, vol. 11, n³. En ligne, consulté le 18 décembre 2018. URL : http://gamestudies.org/1103/articles/sicart_ap.

ThÉRIAULt J.-Y. (2007), Faire société : société civile et espaces francophones, Sudbury, Prise de parole (« Agora»).

Weiner M. (dir.) (2004), Race, Ethnicity and Migration in Modern Japan, Londres/New York, RoutledgeCurzon (« RoutledgeCurzon library of modern Japan »), t. I. 